\title{
Spin Seebeck effect and phonon energy transfer in heterostructures containing layers of a normal metal and a ferromagnetic insulator
}

\author{
A. I. Bezuglyj, ${ }^{1,2, *}$ V. A. Shklovskij, ${ }^{2, \dagger}$ V. V. Kruglyak ${ }^{3}{ }^{2}$ and R. V. Vovk ${ }^{2}$ \\ ${ }^{1}$ National Science Center Kharkov Institute of Physics and Technology, 1, Akademicheskaya St., Kharkov 61108, Ukraine \\ ${ }^{2}$ Kharkov National University, 4, Svobody Sq., Kharkov 61022, Ukraine \\ ${ }^{3}$ School of Physics and Astronomy, University of Exeter EX4 4QL, United Kingdom
}

(Received 19 October 2018; revised manuscript received 2 April 2019; published 19 April 2019)

\begin{abstract}
In the framework of the kinetic approach based on the Boltzmann equation for the phonon distribution function, we analyze phonon heat transfer in a heterostructure containing a layer of a normal metal $(N)$ and a layer of a ferromagnetic insulator $(F)$. Two realistic methods for creating a temperature gradient in such a heterostructure are considered: by heating the $N$ layer by an electric current and by placing the $N / F$ bilayer between massive dielectrics with different temperatures. The electron temperature $T_{e}$ in the $N$ layer and the magnon temperature $T_{m}$ in the $F$ layer are calculated. The difference in these temperatures determines the voltage $V_{\text {ISHE }}$ on the $N$ layer in the Seebeck spin effect regime. The dependence of $V_{\text {ISHE }}$ on the bath temperature and on the thickness of the $N$ and $F$ layers is compared with the available experimental data.
\end{abstract}

DOI: 10.1103/PhysRevB.99.134428

\section{INTRODUCTION}

In recent years, the relation between the processes of spin and heat transport, that is, spin caloritronics, has been of great interest [1-4]. This interest is largely due to the recent observation of the spin Seebeck effect (SSE) in heterostructures containing layers of a normal metal $(N)$ and a ferromagnetic insulator or semiconductor $(F)$, where heat is transferred mainly by phonons [5-9]. Similar to the conventional Seebeck effect, when an electron current emerges as a result of a temperature gradient, the temperature gradient in the SSE generates a spin current. Since it is not yet possible to directly measure the spin current experimentally, a two-layer ferromagnet/normal metal heterostructure $(F / N)$ is used to detect the SSE. In such a structure, the spin current from the $F$ layer is injected into the $N$ metal, where it induces the experimentally observed voltage $V_{\text {ISHE }}$ due to the inverse spin Hall effect (ISHE) [10-14].

Most SSE experiments are carried out in the longitudinal (LSSE) geometry, in which the temperature gradient $\nabla T$ and the spin current $\mathbf{J}_{s}$ are parallel to each other and are oriented perpendicular to the $F / N$ interface [15-17]. In this geometry, to exclude the anomalous voltage in the $F$ layer (due to the Nernst effect [18]), the $F$ layer must be nonconducting, i.e., a magnetic insulator. Currently, in most of the LSSE experiments, yttrium-iron garnet (YIG) is used as a magnetic insulator, and the $N$ layer is made of metals with strong spin-orbit interaction, such as platinum $(\mathrm{Pt})$ or gold $(\mathrm{Au})$.

It is important for us that, in the LSSE experiment, the temperature gradient can be realized in different ways. If thermal sources and sinks with different temperatures are used at the boundaries of the $F / N$ sample, then a good

\footnotetext{
*bezuglyj@kipt.kharkov.ua

†shklovskij@univer.kharkov.ua
}

thermal contact with such thermal reservoirs (thermostats) is needed to create a large temperature gradient [5-7]. When the free surface of the heterostructure is irradiated by a laser beam, the heating occurs locally in a rather narrow region of the sample. The temperature gradients obtained in this way can be described quantitatively only by numerical simulation of temperature profiles [17]. In Refs. [19,20], another very simple method is presented for creating large temperature gradients perpendicular to the $F / N$ interface: by heating the $N$ metal in the $F / N$ bilayer by an electric current. In this case, the $N$ layer used to detect LSSE is simultaneously used both as a resistive heater and a thermometer. In this paper, we analytically consider two ways of realizing the temperature gradient in the LSSE experiment: (a) by heating the $N$ layer with an electric current and (b) by means of two thermostats with different temperatures.

Our investigation of the phonon energy transfer in $F / N$ heterostructures was mainly motivated by the results from Refs. [16,21], where the influence of the thickness and interface of YIG films on the low-temperature increase of the electric signal in the $N$ layer $(\mathrm{Pt})$ under the conditions of the LSSE effect is discussed. When comparing theoretical results with experiment, one should keep in mind that there are two different mechanisms for generating a spin current by a heat flux. The first of them is connected with the difference between the electron and magnon temperatures on the $N / F$ interface. The second mechanism stems from the magnon temperature gradient in the ferromagnetic insulator. At bath temperatures $T_{B}$ significantly lower than the Debye temperature $\Theta_{D}$, the first mechanism will dominate due to the two reasons: (a) the Kapitza thermal resistance of the $N / F$ boundary $R_{\mathrm{th}}$ increases as $T_{B}^{-3}$ [22] and (b) the thermal conductivity of the $F$-plate strongly increases [23]. The latter reason allows us to consider the magnon temperature $T_{m}=$ const, i.e., to neglect the gradient of $T_{m}$ at low temperatures. In this context, we would like to quote the results of Ref. [24], 
where LSSE measurements on a picosecond timescale have been reported. In Ref. [24], it was shown that the spin current $J_{S} \approx \alpha_{S}\left(T_{e}-T_{m}\right)$, where $T_{e}$ is the electron temperature in the $N$ layer. Coefficient $\alpha_{S}$ monotonically decreases with the temperature and vanishes approximately at Curie temperature $\Theta_{C}$ for YIG, as expected from the temperature dependence of Kapitza interfacial resistance. Also, from the independence of $\alpha_{S}$ of thickness of the magnetic insulator (for 20- to 250nm-thick YIG samples), it follows that the contribution from the bulk LSSE is negligible on picosecond timescales. So, in Ref. [24], the interfacial mechanism of LSSE has been separated from bulk LSSE contribution with $\nabla T_{m} \neq 0$. As the generation of the spin current by a temperature difference $T_{e}-T_{m}$ can arise due to heating of the $N$ layer by an ultrashort (femtosecond) laser pulse, the reason for this generation is that at such short times the gradient of the magnon temperature does not have time to form.

Concerning the theoretical ideas explaining the temperature-dependent effective propagation length of thermally excited magnons in YIG introduced in Ref. [16] to explain the dimensionally dependent LSSE responses, one can refer to recent work [25]. In Ref. [25], it was shown that, for the ferrodielectric-insulator interface $(F / I)$ at low temperatures $\left(T \ll \Theta_{D}\right)$, there exists a size effect. The latter manifests itself in the dependence of the Kapitza resistance $R_{\text {th }}$ for thin $F$ plates (films) on the frequency of magnon-phonon collisions, whereas for thick plates the value of $R_{\mathrm{th}}$ does not contain the magnetic characteristics of the ferrodielectric. To explain the growth of the magnetic contribution with decreasing thickness of the $F$ layer, we note that the transfer of heat from the heated magnons to the cooler $I$ layer is provided by phonons. If the thickness of the $F$ layer $d_{f}$ is much larger than the average free path of phonons with respect to their scattering on magnons, $l_{p m}\left(T_{m}\right)$, then the magnons and phonons in the $F$ layer are thermalized and $R_{\mathrm{th}}$ is determined by the acoustic transparency of the $F / I$ interface. In this case, the contribution of magnons to $R_{\mathrm{th}}$ is absent. However, if $d_{f} \ll l_{p m}\left(T_{m}\right)$, then most phonons emitted by magnons in the film leave it without interacting with the magnons, even after several reflections from the boundaries. As a result, in contrast to the case $d_{f} \gg l_{p m}\left(T_{m}\right)$, the Kapitza resistance $R_{\text {th }}$ depends more on the magnon-phonon interaction than on the acoustic transparency of the $F / I$ boundary.

In this paper, we present the kinetic calculations of phonon energy transfer in $N / F / I$ and $I_{1} / N / F / I_{2}$ heterostructures in the SSE regime. The purpose of the work is to find the temperature difference $T_{e}-T_{m}$ between electrons in the $N$ layer and magnons in the $F$ layer, since at low temperatures the difference $T_{e}-T_{m}$ determines the voltage $V_{\mathrm{ISHE}}$ on the $N$ layer.

The paper is organized as follows. In the Introduction (Sec. I), the formulation of the problem of the LSSE in spin caloritronics has been discussed, and some of the most important and relevant studies have been reviewed. In Sec. II, the problem of the temperature profile of the $N / F / I$ heterostructure is solved for a continuous heating of the metal layer and a temperature difference between $F$ and $N$ layers is found for their interface, since this temperature difference determines the strength of the LSSE response. The limiting cases of thin and thick $F$ and $N$ layers are considered. In Sec. III, the

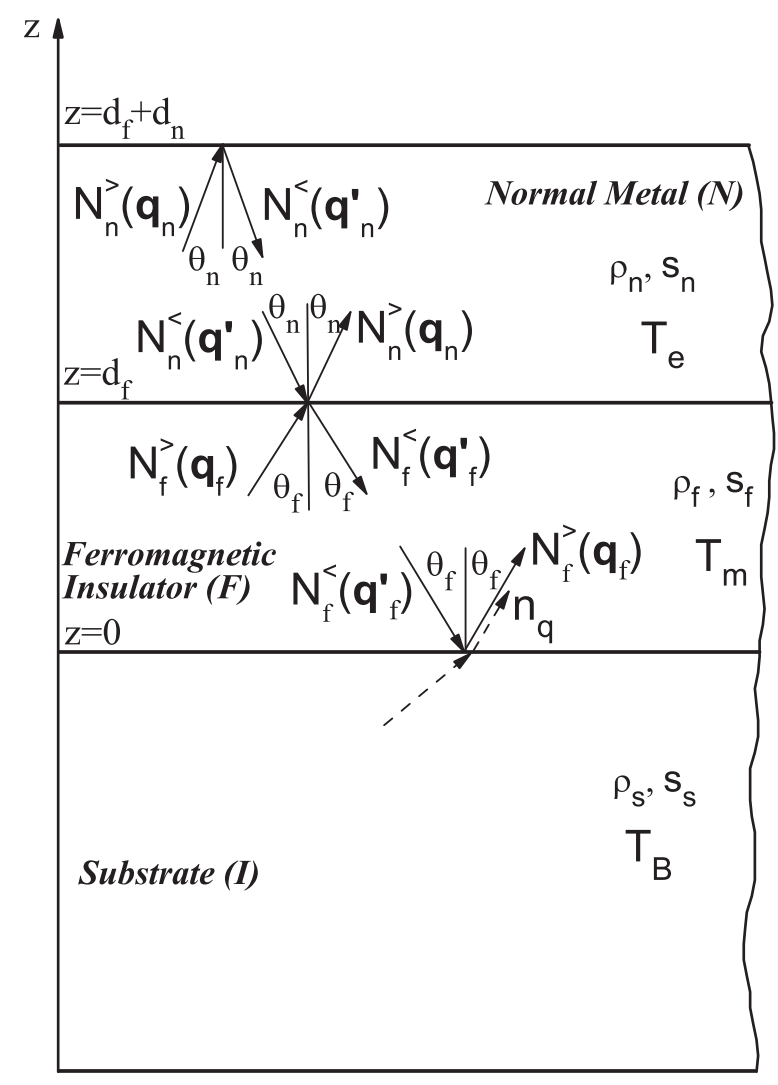

FIG. 1. Refraction and reflection of phonon modes at layer boundaries in the $N / F / I$ structure. The occupation numbers of phonon states with wave vectors $\mathbf{q}$ are denoted by $N \lessgtr(\mathbf{q})$. The superscript $>$ marks phonons with positive $z$ component of $\mathbf{q}$, and the superscript $<$ marks phonons with negative $z$ component of q. The letters $\rho$ and $s$ denote the densities and velocities of the longitudinal sound of the corresponding media. $T_{e}$ is the electron temperature and $T_{m}$ is the magnon temperature. The temperature $T_{B}$ is the temperature of the massive substrate, which plays the role of a thermostat.

heterostructure $I_{1} / N / F / I_{2}$ is investigated, provided that the temperatures of the insulators $I_{1}$ and $I_{2}$ are fixed. As in Sec. II, the cases of thin and thick $F$ and $N$ layers are considered. In Sec. IV, the theory is compared with experiments. The main conclusions of our analytical calculations are formulated in Sec. V. Appendix A contains cumbersome expressions for the coefficients that determine the phonon distribution functions for the $N / F / I$ and $I_{1} / N / F / I_{2}$ layered structures. Appendix B presents the conditions for the applicability of a microscopic model that is used in our paper.

\section{DIFFERENCE BETWEEN THE ELECTRON AND MAGNON TEMPERATURES WITH JOULE HEATING OF ELECTRONS}

\section{A. The problem of heat transfer in a multilayer heterostructure}

A layered structure $N / F / I$ consisting of a metal layer $N$ of thickness $d_{n}$ and a ferromagnetic insulator $F$ of thickness $d_{f}$ (see Fig. 1) is considered. The $F$ layer is in direct contact with a massive dielectric substrate $I$, which plays the role of a thermostat with temperature $T_{B}$. 
To analyze the low-temperature kinetics of electrons and magnons in this structure, we use the following microscopic model. We assume that the energy spectrum of the electrons in the metal is quadratic and isotropic: $\epsilon(\mathbf{p})=p^{2} /(2 m)$, where $\mathbf{p}$ is the quasimomentum of the electron and $m$ is its effective mass. At low temperatures, the dispersion law of phonons can be approximated by the linear relation $\omega_{q i}=s_{i} q_{i}$, where $q_{i}$ is the absolute value of the phonon wave vector and $s_{i}$ is the longitudinal sound velocity in the corresponding medium $(i=n, f, s)$. Here and below, we take into account only the longitudinal acoustic branch of lattice vibrations. Taking into account the transverse vibration modes does not change the physical picture of the energy relaxation in the layered structure. Yet, the inter conversion of the phonon modes with different polarizations at the interlayer boundaries complicates the description so much that an analytical approach becomes formidable (see the discussion in Refs. [26,27]). The transfer of energy between electrons and phonons is a consequence of the electron-phonon interaction, for which we use the deformation potential approximation. The interaction of magnons with the lattice in the magnon temperature approximation was analyzed in Ref. [25], the results of which will be used below. The energy exchange between layers that have a phonon nature will be described in terms of the well-known model of the acoustic mismatch [22,28,29].

Suppose that the electrons in the $N$ layer are thermalized and have a temperature $T_{e}$. We also assume that the magnons are thermalized in the $F$ layer and their temperature is $T_{m}$. In addition, we assume that, due to the high electron and magnon thermal conductivities [23], the temperatures $T_{e}$ and $T_{m}$ do not depend on the coordinate $z$ perpendicular to the layers, that is, they are constants. In contrast, the phonon distribution functions in the $N$ and $F$ layers depend on $z$ and obey the kinetic equations. For the $N$ layer, we have

$$
s_{n z} \frac{d N_{n}\left(\mathbf{q}_{n}, z\right)}{d z}=I_{p e}\left[N_{n}\left(\mathbf{q}_{n}, z\right)\right],
$$

where $s_{n z}$ is the projection of the phonon velocity on the $z$ axis, and $I_{p e}$ is the phonon-electron collision integral, which in the case of the Fermi electron distribution function has a simple form [30]:

$$
I_{p e}\left[N_{n}\left(\mathbf{q}_{n}, z\right)\right]=v_{n}(q)\left[n_{q}\left(T_{e}\right)-N_{n}\left(\mathbf{q}_{n}, z\right)\right] .
$$

Here $n_{q}\left(T_{e}\right)=\left[\exp \left(\hbar \omega_{q n} / T_{e}\right)-1\right]^{-1}$ is the Bose distribution function $\left(k_{B}=1\right)$. In the approximation of the deformation potential, the frequency of phonon-electron collisions is given by

$$
v_{n}=\frac{m^{2} \mu^{2}}{2 \pi \hbar^{3} \rho_{n} s_{n}} \omega_{q n},
$$

where $\mu$ is the deformation potential constant, which is of the order of the Fermi energy $\epsilon_{F} ; \rho_{n}$ is the density of the metal. Note that Eq. (3) refers to the case of a pure metal. In dirty metals, the dependence of $v_{n}$ on $\omega_{q n}$ can be approximated by a power function with a power exponent that depends on the type of defects [31]. The phonon distribution function in the magnetic layer obeys the kinetic equation

$$
s_{f z} \frac{d N_{f}\left(\mathbf{q}_{f}, z\right)}{d z}=I_{p m}\left[N_{f}\left(\mathbf{q}_{f}, z\right)\right],
$$

where the collision integral of phonons with magnons has the form [25]

$$
I_{p m}\left[N_{f}\left(\mathbf{q}_{f}, z\right)\right]=v_{f}\left(T_{m}, q\right)\left[n_{q}\left(T_{f}\right)-N_{f}\left(\mathbf{q}_{f}, z\right)\right] .
$$

In contrast to the phonon-electron collision frequency, which depends only on the absolute value of the wave vector of the phonon $q$, the frequency of phonon-magnon collisions also depends on the temperature of the magnons $T_{m}$. For the phonon-magnon collision frequency, as it was shown in Ref. [25],

$$
v_{f}\left(T_{m}, q\right)=D\left(T_{m}\right) I\left(T_{m}, q\right),
$$

where

$$
\begin{gathered}
D\left(T_{m}\right)=\frac{\Theta_{C}}{8 \pi M_{f} a_{f} s_{f}}\left(\frac{T_{m}}{\Theta_{C}}\right)^{3}, \\
I\left(T_{m}, q\right)=\int_{y_{0}}^{\infty} d y y(x+y)\left(\frac{1}{e^{y}-1}-\frac{1}{e^{x+y}-1}\right) .
\end{gathered}
$$

We note that $I\left(T_{m}, q\right)$ contains the dependence on the phonon wave vector through the dimensionless value $x=\hbar \omega_{q f} / T_{m}$. In Eq. (7), $\Theta_{C}$ is the Curie temperature, $M_{f}$ is the mass of the magnetic atom, $a_{f}$ is the lattice constant of the ferromagnet. In Eq. (8), the lower limit of integration, $y_{0}=\frac{\Theta_{D f}^{2}}{4 T_{m} \Theta_{C}}$, accounts for that the emission of a phonon by a magnon is possible only when the magnon energy is greater than $\frac{\Theta_{D f}^{2}}{4 \Theta_{C}}$. Here, $\Theta_{D f}$ is the Debye temperature of the ferromagnet.

To formulate the boundary conditions, we turn to Fig. 1, which illustrates the processes of reflection and refraction of phonons at layer boundaries. Figure 1 shows that, in the ferromagnet $F$ near the boundary with the substrate, the distribution function of phonons having a positive $z$ component of the wave vector contains two contributions. One of them is determined by phonons coming from the substrate, and the other is due to the phonons of the ferromagnet, reflected from the boundary:

$$
N_{f}^{>}\left(\mathbf{q}_{f}, 0\right)=\alpha_{s \rightarrow f}\left(\theta_{s}\right) n_{q}\left(T_{B}\right)+\beta_{f \rightarrow s}\left(\theta_{f}\right) N_{f}^{<}\left(\mathbf{q}_{f}^{\prime}, 0\right) .
$$

Hereinafter, the Greek letters $\alpha$ and $\beta$ denote the probability of passing a phonon through the boundary between adjacent materials and the probability of reflection from the boundary $(\beta=1-\alpha)$. The subscripts in $\alpha$ and $\beta$ define the boundary. $T_{B}$ is the temperature of the substrate. Everywhere in the boundary conditions, the wave vectors $\mathbf{q}$ and $\mathbf{q}^{\prime}$ represent phonons that have a positive or negative $z$ component of the wave vector: $\mathbf{q}=\left(q_{x}, q_{y}, q_{z}>0\right), \mathbf{q}^{\prime}=\left(q_{x}, q_{y}, q_{z}<0\right)$. Correspondingly, $N^{>}$and $N^{<}$denote the occupation numbers of states with wave vectors $\mathbf{q}$ and $\mathbf{q}^{\prime}$.

The condition Eq. (9) assumes that the phonons that have flown from the $F$ layer to the substrate do not return back. Such a picture is typical for single-crystal substrates with high thermal conductivity, where phonons propagate ballistically.

In the acoustic mismatch model [22,28,29], the probability of passing the interface depends on the angle of incidence of the phonon $\theta$ and the acoustic impedances of the adjacent media $Z=\rho s$ and $Z^{\prime}=\rho^{\prime} s^{\prime}$ :

$$
\alpha(\theta)=\frac{4 Z Z^{\prime} \cos \theta \cos \theta^{\prime}}{\left(Z \cos \theta^{\prime}+Z^{\prime} \cos \theta\right)^{2}} .
$$


The angles of incidence and refraction are related by $\sin \theta=$ $\left(s / s^{\prime}\right) \sin \theta^{\prime}$. Here, adjacent materials are characterized by quantities with a prime and without it.

Conditions on the boundaries $z=d_{f}$ and $z=d_{f}+d_{n}$ are written analogously to the relation Eq. (9). For $z=d_{f}$, we have

$$
\begin{aligned}
N_{f}^{<}\left(\mathbf{q}_{f}^{\prime}, d_{f}\right)= & \alpha_{n \rightarrow f}\left(\theta_{n}\right) N_{n}^{<}\left(\mathbf{q}_{n}^{\prime}, d_{f}\right) \\
& +\beta_{f \rightarrow n}\left(\theta_{f}\right) N_{f}^{>}\left(\mathbf{q}_{f}, d_{f}\right), \\
N_{n}^{>}\left(\mathbf{q}_{n}, d_{f}\right)= & \alpha_{f \rightarrow n}\left(\theta_{f}\right) N_{f}^{>}\left(\mathbf{q}_{f}^{\prime}, d_{f}\right) \\
& +\beta_{n \rightarrow f}\left(\theta_{n}\right) N_{n}^{<}\left(\mathbf{q}_{n}, d_{f}\right) .
\end{aligned}
$$

It follows from Eq. (10) that $\alpha_{n \rightarrow f}\left(\theta_{n}\right)=\alpha_{f \rightarrow n}\left(\theta_{f}\right)$.

For $z=d_{f}+d_{n}$, the boundary condition describes the specular reflection of phonons at the outer boundary of the metallic layer:

$$
N_{n}^{>}\left(\mathbf{q}_{n}, d_{f}+d_{n}\right)=N_{n}^{<}\left(\mathbf{q}_{n}^{\prime}, d_{f}+d_{n}\right) .
$$

\section{B. Analytical solution of the heat transfer problem in a layered $N / F / I$-system}

The purpose of our calculations is to find the temperature difference $T_{e}-T_{m}$, which appears in the expression for the voltage on a normal metal under the conditions of the SSE $[17,32]$. This difference is determined by the heat flux $Q$ from a normal metal heated by an electric current to a cooler dielectric substrate. Since the transfer of heat through the $N / F / I$ system is of a phonon nature, to calculate $T_{e}-T_{m}$, it is necessary to solve the kinetic equations for the phonon distribution function in $N$ and $F$ layers and to stitch the solutions at the interfaces. The solutions of these kinetic equations have the form

$$
N_{i}^{>}\left(\mathbf{q}_{i}, z\right)=C_{i}^{>}\left(\mathbf{q}_{i}\right) \exp \left(-z / l_{i}\right)+n_{q_{i}}\left(T_{i}\right),
$$

for $q_{z}>0$ and

$$
N_{i}^{<}\left(\mathbf{q}_{i}^{\prime}, z\right)=C_{i}^{<}\left(\mathbf{q}_{i}^{\prime}\right) \exp \left(z / l_{i}\right)+n_{q_{i}}\left(T_{i}\right),
$$

for $q_{z}<0$. Here $l_{i}=\left|s_{i z}\right| / v_{q i}$, where the index $i$ takes values $n$ or $f$. The coefficients $C_{i}^{\gtrless}$ are calculated in Appendix A.

The equations for the electron temperature in the metallic layer and the magnon temperature in the magnetic layer are determined by the thermal balance conditions in the corresponding layers: $P_{e p}=W$ and $P_{m p}=0$, where $W$ is the specific power of the heat sources in the $N$ layer and $P_{e p}$ and $P_{m p}$ are the specific powers (averaged over the layer thickness), that are transferred from electrons to phonons and from magnons to phonons, respectively. $P_{e p}$ is expressed in terms of the phonon-electron collision integral as

$$
P_{e p}=\frac{1}{d_{n}} \int_{d_{f}<z<d_{n}+d_{f}} d z \int \frac{d^{3} q}{(2 \pi)^{3}} \hbar \omega_{q} I_{p e}\left[N_{n}\left(\mathbf{q}_{n}, z\right)\right] .
$$

The substitution of Eqs. (14) and (15) yields for the $N$ layer

$$
\begin{aligned}
W= & \frac{1}{d_{n}} \int_{q_{z}>0} \frac{d^{3} q}{(2 \pi)^{3}} \hbar \omega_{n q} s_{n z}\left[C_{n}^{>} e^{-d_{f} / l_{n}}\left(e^{-d_{n} / l_{n}}-1\right)\right. \\
& \left.-C_{n}^{<} e^{d_{f} / l_{n}}\left(e^{d_{n} / l_{n}}-1\right)\right],
\end{aligned}
$$

and for the $F$ layer we obtain

$$
\int_{q_{z}>0} \frac{d^{3} q}{(2 \pi)^{3}} \hbar \omega_{f q} s_{f z}\left[C_{f}^{>}\left(e^{-d_{f} / l_{f}}-1\right)-C_{f}^{<}\left(e^{d_{f} / l_{f}}-1\right)\right]=0 .
$$

Taking into account the explicit form of the coefficients $C_{i}^{\gtrless}$, we have

$$
\begin{aligned}
W= & \frac{1}{d_{n}} \int_{q_{z}>0} \frac{d^{3} q}{(2 \pi)^{3}} \hbar \omega_{n q} s_{n z}\left(e^{2 d_{n} / l_{n}}-1\right) \frac{\alpha_{2}}{D} \\
& \times\left\{\alpha_{1} e^{d_{f} / l_{f}}\left[n_{q}\left(T_{m}\right)-n_{q}\left(T_{B}\right)\right]+\left(e^{2 d_{f} / l_{f}}-\beta_{1}\right)\right. \\
& \left.\times\left[n_{q}\left(T_{e}\right)-n_{q}\left(T_{m}\right)\right]\right\}
\end{aligned}
$$

and

$$
\begin{aligned}
& \int_{q_{z}>0} \frac{d^{3} q}{(2 \pi)^{3}} \hbar \omega_{f q} s_{f z} \frac{1}{D}\left(e^{d_{f} / l_{f}}-1\right)\left\{\alpha _ { 1 } \left[e^{2 d_{n} / l_{n}}\left(e^{d_{f} / l_{f}}+\beta_{2}\right)\right.\right. \\
& \left.\quad-\beta_{2} e^{d_{f} / l_{f}}-\beta_{2}+\alpha_{2}\right]\left[n_{q}\left(T_{m}\right)-n_{q}\left(T_{B}\right)\right] \\
& \left.\quad-\alpha_{2}\left(e^{2 d_{n} / l_{n}}-1\right)\left(e^{d_{f} / l_{f}}+\beta_{1}\right)\left[n_{q}\left(T_{e}\right)-n_{q}\left(T_{m}\right)\right]\right\}=0 .
\end{aligned}
$$

In the case of thick $F$ and $N$ layers, when $d_{f} \gg l_{f}$ and $d_{n} \gg l_{n}$, Eq. (19) is greatly simplified and reduces to

$$
\begin{aligned}
W= & \frac{1}{d_{n}} \int_{0}^{\infty} \frac{q^{2} d q}{(2 \pi)^{2}} \int_{0}^{\pi / 2} \sin \theta d \theta \cos \theta \alpha_{2}(\theta) s_{n} \hbar \omega_{q} \\
& \times\left[n_{q}\left(T_{e}\right)-n_{q}\left(T_{m}\right)\right] .
\end{aligned}
$$

If we introduce the probability $\alpha_{2}$ averaged over the angles of incidence,

$$
\left\langle\alpha_{2}\right\rangle=2 \int_{0}^{\pi / 2} \sin \theta d \theta \cos \theta \alpha_{2}(\theta),
$$

then integration over the phonon wave vectors gives

$$
W d_{n} \equiv Q=\frac{\pi^{2}\left\langle\alpha_{2}\right\rangle}{120 \hbar^{3} s_{n}^{2}}\left(T_{e}^{4}-T_{m}^{4}\right) .
$$

For weak heating, when $T_{e}-T_{B} \ll T_{B}$ and $T_{m}-T_{B} \ll T_{B}$, from Eq. (23) we obtain the temperature difference on the $N / F$ boundary as

$$
T_{e}-T_{m}=\frac{30 \hbar^{3} s_{n}^{2}}{\pi^{2}\left\langle\alpha_{2}\right\rangle} \frac{W d_{n}}{T_{B}^{3}}
$$

We now consider the case of thin layers, when $d_{f} \ll l_{f}$ and $d_{n} \ll l_{n}$. In this limit, the determinant $D=\alpha_{1} \alpha_{2}$, and this leads to the following equation for the electron temperature:

$$
W=\int_{q_{z}>0} \frac{d^{3} q}{(2 \pi)^{3}} \hbar \omega_{q n} v_{q n}\left[n_{q}\left(T_{e}\right)-n_{q}\left(T_{B}\right)\right] .
$$

Equation (25) exactly coincides with the equation for the electron temperature in a thin metal film lying on a dielectric substrate (see, for example, [31]). The integral over the phonon wave vectors gives

$$
W=\Sigma_{5}\left(T_{e}^{5}-T_{B}^{5}\right),
$$


where the electron-phonon coupling constant is

$$
\Sigma_{5}=\frac{D_{5} m^{2} \mu^{2}}{4 \pi^{3} \rho \hbar^{7} s^{4}} .
$$

The number $D_{5} \approx 24.9$ is the integral $D_{k}=\int_{0}^{\infty} x^{k-1}\left(e^{x}-\right.$ $1)^{-1} d x$ for $k=5$. As can be seen from Eq. (26), a thin layer of a ferromagnet has no effect on the kinetics of nonequilibrium phonons, since they are almost not absorbed in it.

The magnon temperature $T_{m}$ in the $F$ layer is determined by Eq. (20). It turns out that in the case of thin $F$ layers $T_{m}=T_{B}$, i.e., the magnon temperature coincides with the temperature of the thermostat. The reason for this is clearthe nonequilibrium phonons emitted in the metallic layer go into the substrate without being absorbed by the magnons in the ferromagnet layer, and therefore, do not heat the magnon gas.

Since in the case of thin $F$ layers $T_{m}=T_{B}$, the temperature difference between electrons and magnons for thin $F$ layers can be obtained from Eq. (26). With weak heating, we have $T_{e}-T_{m}=W / 5 \Sigma_{5} T_{B}^{4}$, from which it follows that the temperature jump on the $N / F$ boundary increases as the temperature of the thermostat $T_{B}$ decreases. Since the voltage on a normal metal under the conditions of both the longitudinal and transverse SSEs is proportional to the difference $T_{e}-T_{m}$ $[17,32]$, this voltage should increase as the temperature of the thermostat is lowered.

Experimental studies of the SSE are often realized on $N / F$ heterostructures in which the $F$ layer has a rather large thickness, whereas the $N$ layer is thin. To apply our results to this case, we consider the limit $d_{f} \gg l_{f}$ and $d_{n} \ll l_{n}$. Then, the magnon temperature is determined by equation

$$
W d_{n}=\frac{\pi^{2}\left\langle\alpha_{1}\right\rangle}{120 \hbar^{3} s_{f}^{2}}\left(T_{m}^{4}-T_{B}^{4}\right) \equiv \Sigma_{4} d_{n}\left(T_{m}^{4}-T_{B}^{4}\right),
$$

and for the electron temperature we have

$$
W=\Sigma_{5}\left(T_{e}^{5}-T_{m}^{5}\right) .
$$

As in the case of thin $F$ layers, the temperature jump on the $N / F$ boundary for weak heating is given by $T_{e}-T_{m}=$ $W / 5 \Sigma_{5} T_{B}^{4}$, i.e., this jump rapidly increases as the temperature of the thermostat $T_{B}$ decreases.

Let us graphically show the dependence of the temperature jump $T_{e}-T_{m}$ on the temperature of the thermostat. In the case of thick $F$ layers, when $d_{f} \gg l_{f}$ (but $d_{n} \ll l_{n}$ ), the temperature difference between electrons and magnons is determined by Eq. (29), and the temperature difference between magnons and thermostat is given by Eq. (28). From these equations it follows that

$$
\frac{T_{e}-T_{m}}{T_{0}}=\left[w+\left(1+t_{B}\right)^{5 / 4}\right]^{1 / 5}-\left(1+t_{B}^{4}\right)^{1 / 4}
$$

Here, the dimensionless temperature of the thermostat is $t_{B}=T_{B} / T_{0} ; T_{0}=\left(W / \Sigma_{4}\right)^{1 / 4}$. The notation $w=\Sigma_{4}^{5 / 4} / \Sigma_{5} W$ is introduced.

In the case when both layers are thin, i.e., $d_{n} \ll l_{n}$ and $d_{f} \ll l_{f}$, the dimensionless difference between the electron and magnon temperatures has the form

$$
\frac{T_{e}-T_{m}}{T_{0}}=\left(w+t_{B}^{5}\right)^{1 / 5}-t_{B} .
$$

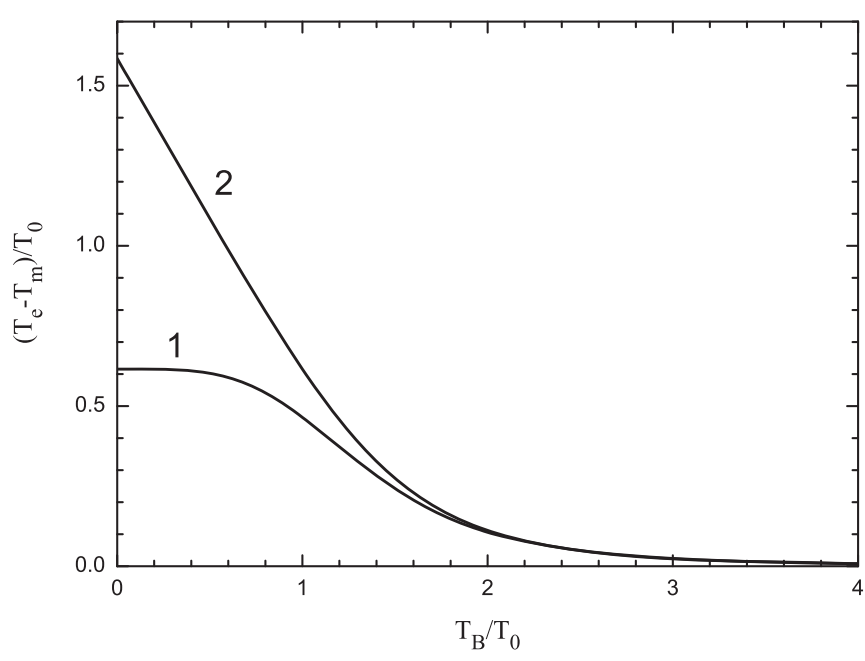

FIG. 2. The dimensionless difference between the electron and magnon temperatures for thick $F$ layers (1) and for thin $F$ layers (2).

The dependencies Eqs. (30) and (31) are shown in Fig. 2.

\section{HEAT TRANSFER THROUGH A TWO-LAYER N/F SYSTEM LOCATED BETWEEN TWO INSULATORS WITH DIFFERENT TEMPERATURES}

Now we consider the layered structure $I_{1} / N / F / I_{2}$ in which a metal layer with a thickness of $d_{n}$ and a layer of a ferromagnetic insulator with a thickness $d_{f}$ are placed between two massive dielectrics $I_{1}$ and $I_{2}$. The temperatures of the dielectrics are $T_{B}$ and $T_{H}$ with $T_{H}>T_{B}$ (see Fig. 3).

As in Sec. II, we assume that the electrons in the $N$ layer and the magnons in the $F$ layer are thermalized and have temperatures $T_{e}$ and $T_{m}$, respectively. Again, we will assume that, owing to the high electron and magnon thermal conductivities, the temperatures $T_{e}$ and $T_{m}$ do not depend on the coordinate $z$ perpendicular to the layers [23]. In contrast, the phonon distribution functions in the $N$ layer and in the $F$ layer depend on $z$, and this dependence is determined by the corresponding kinetic equations. For the $N$ layer, the kinetic equation has form Eq. (1), and for the $F$ layer it has form $\mathrm{Eq}$, (4). Each of these equations can be subdivided into two equations by writing them for $N^{>}(\mathbf{q}, z)$ and $N^{<}\left(\mathbf{q}^{\prime}, z\right)$, that is for the distribution functions of the phonons with positive and negative projections of the wave vector on the $z$ axis. To find the phonon distribution functions, the boundary conditions must be added to Eqs. (1) and (4). For the boundaries $z=0$ and $z=d_{f}$, they do not differ from the expressions Eqs. (9), (11), and (12) given in Sec. II:

$$
\begin{gathered}
N_{f}^{>}\left(\mathbf{q}_{f}, 0\right)=\alpha_{1} n_{q}\left(T_{B}\right)+\beta_{1} N_{f}^{<}\left(\mathbf{q}_{f}^{\prime}, 0\right), \\
N_{f}^{<}\left(\mathbf{q}_{f}^{\prime}, d_{f}\right)=\alpha_{2} N_{n}^{<}\left(\mathbf{q}_{n}^{\prime}, d_{f}\right)+\beta_{2} N_{f}^{>}\left(\mathbf{q}_{f}, d_{f}\right), \\
N_{n}^{>}\left(\mathbf{q}_{n}, d_{f}\right)=\alpha_{2} N_{f}^{>}\left(\mathbf{q}_{f}^{\prime}, d_{f}\right)+\beta_{2} N_{n}^{<}\left(\mathbf{q}_{n}, d_{f}\right) .
\end{gathered}
$$

For $z=d_{f}+d_{n}$, the boundary condition has the same structure as for $z=0$ :

$$
N_{n}^{<}\left(\mathbf{q}_{n}^{\prime}, d_{f}+d_{n}\right)=\beta_{3} N_{n}^{>}\left(\mathbf{q}_{n}, d_{f}+d_{n}\right)+\alpha_{3} n_{q}\left(T_{H}\right) .
$$




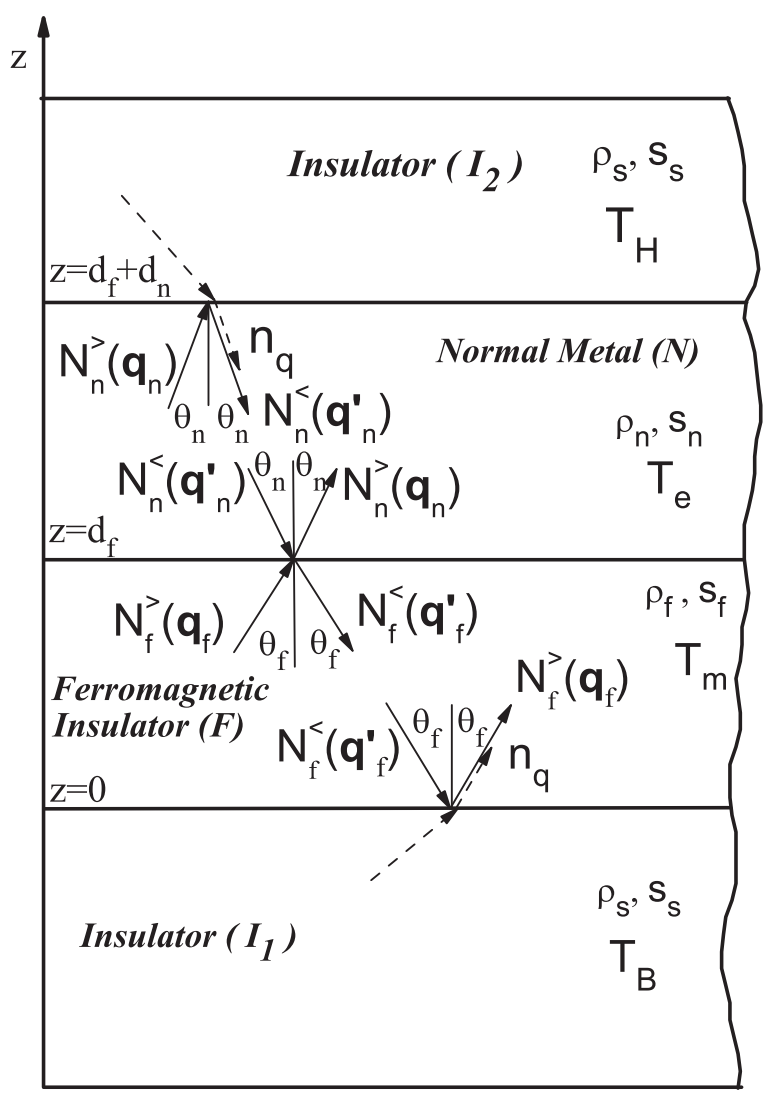

FIG. 3. Refraction and reflection of phonon modes at interfaces in a layered $I_{1} / N / F / I_{2}$ structure. $I_{1}$ is a massive dielectric substrate (heat sink with temperature $T_{B}$ ), $F$ is a ferromagnetic insulator, $N$ is a normal metal, $I_{2}$ is a heat source in the form of a thick dielectric layer with temperature $T_{H}>T_{B}$. The remaining notations are the same as in Fig. 1.

Substitution of the solutions Eqs. (14) and (15) into the boundary conditions Eqs. (32), (33), (34) and (35) gives the coefficients $C \gtrless$ (see Appendix A).

To find the temperatures $T_{e}$ and $T_{m}$, we use the condition that on all interfaces the heat flux is the same. If we denote the $z$ component of the heat flux as $Q_{z}(z)$, then the equations for $T_{e}$ and $T_{m}$ can be written as

$$
\begin{gathered}
Q_{z}\left(d_{f}-0\right)=Q_{z}(+0), \\
Q_{z}\left(s=d_{f}+d_{n}-0\right)=Q_{z}\left(d_{f}+0\right) .
\end{gathered}
$$

The $z$ component of the phonon heat flux in the $N$ layer is expressed in terms of the phonon distribution function as

$$
Q_{z}=\int_{q_{z}>0} \frac{d^{3} q}{(2 \pi)^{3}} \hbar \omega_{q} s_{n z}\left[N_{n}^{>}\left(\mathbf{q}_{n}, z\right)-N_{n}^{<}\left(\mathbf{q}_{n}^{\prime}, z\right)\right]
$$

The expression for the phonon heat flux in the $F$ layer has a completely similar form. Note that the equality $Q_{z}\left(d_{f}-0\right)=$ $Q_{z}\left(d_{f}+0\right)$ is automatically satisfied (see Ref. [22]).

Substitution of the phonon distribution functions into
Eq. (36) gives

$$
\begin{gathered}
\int_{q_{z}>0} \frac{d^{3} q}{(2 \pi)^{3}} \hbar \omega_{q} s_{f z} \frac{\left(e^{d_{f} / l_{f}}-1\right)}{D}\left\{\alpha_{2} \alpha_{3} e^{d_{n} / l_{n}}\left(e^{d_{f} / l_{f}}+\beta_{1}\right)\right. \\
\quad \times\left[n_{q}\left(T_{H}\right)-n_{q}\left(T_{e}\right)\right]+\alpha_{2}\left(e^{2 d_{n} / l_{n}}-\beta_{3}\right)\left(e^{d_{f} / l_{f}}+\beta_{1}\right) \\
\times\left[n_{q}\left(T_{e}\right)-n_{q}\left(T_{m}\right)\right]-\alpha_{1}\left(e^{2 d_{n} / l_{n}+d_{f} / l_{f}}-\beta_{2} \beta_{3} e^{d_{f} / l_{f}}\right. \\
\left.\left.+\beta_{2} e^{2 d_{n} / l_{n}}-\left(\beta_{2}-\alpha_{2}\right) \beta_{3}\right)\left[n_{q}\left(T_{m}\right)-n_{q}\left(T_{B}\right)\right]\right\}=0
\end{gathered}
$$

After a similar substitution of the corresponding phonon distribution functions into the Eq. (37), we obtain

$$
\begin{gathered}
\int_{q_{z}>0} \frac{d^{3} q}{(2 \pi)^{3}} \hbar \omega_{q} s_{n z} \frac{\left(e^{d_{n} / l_{n}}-1\right)}{D}\left\{\alpha_{1} \alpha_{2} e^{d_{f} / l_{f}}\left(e^{d_{n} / l_{n}}+\beta_{3}\right)\right. \\
\times\left[n_{q}\left(T_{m}\right)-n_{q}\left(T_{B}\right)\right]+\alpha_{2}\left(e^{2 d_{f} / l_{f}}-\beta_{1}\right)\left(e^{d_{n} / l_{n}}+\beta_{3}\right) \\
\times\left[n_{q}\left(T_{e}\right)-n_{q}\left(T_{m}\right)\right]-\alpha_{3}\left(e^{2 d_{f} / l_{f}+d_{n} / l_{n}}-\beta_{1} \beta_{2} e^{d_{n} / l_{n}}\right. \\
\left.\left.+\beta_{2} e^{2 d_{f} / l_{f}}-\beta_{1}\left(\beta_{2}-\alpha_{2}\right)\right)\left[n_{q}\left(T_{H}\right)-n_{q}\left(T_{e}\right)\right]\right\}=0 .
\end{gathered}
$$

In the case of thick layers $\left(d_{n} \gg l_{n}, d_{f} \gg l_{f}\right)$, the equations for $T_{e}$ and $T_{m}$ have the form

$$
\begin{aligned}
& \int_{q_{z}>0} \frac{d^{3} q}{(2 \pi)^{3}} \hbar \omega_{q} s_{f z}\left\{\alpha_{2}\left[n_{q}\left(T_{e}\right)-n_{q}\left(T_{m}\right)\right]\right. \\
& \left.\quad-\alpha_{1}\left[n_{q}\left(T_{m}\right)-n_{q}\left(T_{B}\right)\right]\right\}=0, \\
& \int_{q_{z}>0} \frac{d^{3} q}{(2 \pi)^{3}} \hbar \omega_{q} s_{n z}\left\{\alpha_{3}\left[n_{q}\left(T_{H}\right)-n_{q}\left(T_{e}\right)\right]\right. \\
& \left.\quad-\alpha_{2}\left[n_{q}\left(T_{e}\right)-n_{q}\left(T_{m}\right)\right]\right\}=0 .
\end{aligned}
$$

Integration over the phonon wave vectors gives

$$
\begin{aligned}
\left\langle\alpha_{2}\right\rangle_{f}\left(T_{e}^{4}-T_{m}^{4}\right) & =\left\langle\alpha_{1}\right\rangle_{f}\left(T_{m}^{4}-T_{B}^{4}\right), \\
\left\langle\alpha_{3}\right\rangle_{n}\left(T_{H}^{4}-T_{e}^{4}\right) & =\left\langle\alpha_{2}\right\rangle_{n}\left(T_{e}^{4}-T_{m}^{4}\right) .
\end{aligned}
$$

As before, the notations $\langle\alpha\rangle_{f}$ and $\langle\alpha\rangle_{n}$ denote the averaging over the angles of phonon incidence $\theta_{f}$ or $\theta_{n}$ of the probability of the phonon passing through the corresponding interlayer boundary. For example,

$$
\left\langle\alpha_{2}\right\rangle_{n}=2 \int_{0}^{\pi / 2} \sin \left(\theta_{n}\right) \cos \left(\theta_{n}\right) \alpha_{2}\left(\theta_{n}\right) d \theta_{n} .
$$

Using Snell's law $s_{n} \sin \left(\theta_{f}\right)=s_{f} \sin \left(\theta_{n}\right)$, one can show that $s_{f}^{2}\left\langle\alpha_{2}\right\rangle_{n}=s_{n}^{2}\left\langle\alpha_{2}\right\rangle_{f}$.

The solutions of the set of Eqs. (43) and (44) are

$$
\begin{aligned}
T_{m}^{4} & =\frac{\left\langle\alpha_{2}\right\rangle_{f}\left\langle\alpha_{3}\right\rangle_{n} T_{H}^{4}+\left\langle\alpha_{1}\right\rangle_{f}\left(\left\langle\alpha_{2}\right\rangle_{n}+\left\langle\alpha_{3}\right\rangle_{n}\right) T_{B}^{4}}{\left\langle\alpha_{1}\right\rangle_{f}\left\langle\alpha_{2}\right\rangle_{n}+\left\langle\alpha_{1}\right\rangle_{f}\left\langle\alpha_{3}\right\rangle_{n}+\left\langle\alpha_{2}\right\rangle_{f}\left\langle\alpha_{3}\right\rangle_{n}}, \\
T_{e}^{4} & =\frac{\left(\left\langle\alpha_{1}\right\rangle_{f}+\left\langle\alpha_{2}\right\rangle_{f}\right)\left\langle\alpha_{3}\right\rangle_{n} T_{H}^{4}+\left\langle\alpha_{1}\right\rangle_{f}\left\langle\alpha_{2}\right\rangle_{n} T_{B}^{4}}{\left\langle\alpha_{1}\right\rangle_{f}\left\langle\alpha_{2}\right\rangle_{n}+\left\langle\alpha_{1}\right\rangle_{f}\left\langle\alpha_{3}\right\rangle_{n}+\left\langle\alpha_{2}\right\rangle_{f}\left\langle\alpha_{3}\right\rangle_{n}} .
\end{aligned}
$$

In the case when the temperatures $T_{H}$ and $T_{B}$ differ only slightly, so that $T_{H}-T_{B} \ll T_{B}$, the temperature difference between electrons and magnons is given by

$$
T_{e}-T_{m}=\frac{\left\langle\alpha_{1}\right\rangle_{f}\left\langle\alpha_{3}\right\rangle_{n}\left(T_{H}-T_{B}\right)}{\left\langle\alpha_{1}\right\rangle_{f}\left\langle\alpha_{2}\right\rangle_{n}+\left\langle\alpha_{1}\right\rangle_{f}\left\langle\alpha_{3}\right\rangle_{n}+\left\langle\alpha_{2}\right\rangle_{f}\left\langle\alpha_{3}\right\rangle_{n}} .
$$


We now turn to the limiting case of thin layers, when $d_{f} \ll$ $l_{f}$ and $d_{n} \ll l_{n}$. In this limit, the equations for $T_{e}$ and $T_{m}$ have the following form:

$$
\begin{aligned}
& \int_{q_{z}>0} \frac{d^{3} q}{(2 \pi)^{3}} \hbar \omega_{q n} v_{q n} \frac{1}{D}\left\{\alpha_{1} \alpha_{2}\left(1+\beta_{3}\right)\left[n_{q}\left(T_{e}\right)-n_{q}\left(T_{B}\right)\right]\right. \\
& \left.-\alpha_{3}\left(1+\beta_{1} \alpha_{2}+\beta_{2} \alpha_{1}-\beta_{1} \beta_{2}\right)\left[n_{q}\left(T_{H}\right)-n_{q}\left(T_{e}\right)\right]\right\}=0 \\
& \int_{q_{z}>0} \frac{d^{3} q}{(2 \pi)^{3}} \hbar \omega_{q f} v_{q f} \frac{1}{D}\left\{\alpha_{2} \alpha_{3}\left(1+\beta_{1}\right)\left[n_{q}\left(T_{H}\right)-n_{q}\left(T_{m}\right)\right]\right. \\
& \left.\quad-\alpha_{1}\left(1+\beta_{2} \alpha_{3}+\beta_{3} \alpha_{2}-\beta_{2} \beta_{3}\right)\left[n_{q}\left(T_{m}\right)-n_{q}\left(T_{B}\right)\right]\right\}=0,
\end{aligned}
$$

where $D=1-\beta_{1} \beta_{2}-\beta_{2} \beta_{3}+\beta_{1}\left(\beta_{2}-\alpha_{2}\right) \beta_{3}$.

It is interesting that, unlike the case of continuous heating of the $N$ layer considered in Sec. II, in the $I_{1} / N / F / I_{2}$ heterostructure with two massive dielectric plates maintained at different temperatures, the electron and magnon temperatures depend on the acoustic transparencies of interfaces even for arbitrarily small layer thicknesses $d_{f}$ and $d_{n}$. This can be easily seen by noting that the layer thicknesses $d_{f}$ and $d_{n}$ drop out of Eqs. (49) and (50).

In the integrals Eqs. (49) and (50), the integration over angles is separated from the integration over the absolute value of the phonon wave vector. Because of the simple linear relationship between $v_{q n}$ and $\omega_{q n}$, the equation for the electron temperature reduces to the following form:

$$
T_{e}^{5}=\frac{\Psi_{31} T_{H}^{5}+\Phi_{13} T_{B}^{5}}{\Psi_{31}+\Phi_{13}}
$$

where we have denoted

$$
\Phi_{13}=\int_{0}^{\pi / 2} d \theta \sin (\theta) \alpha_{1} \alpha_{2}\left(1+\beta_{3}\right) / D
$$

and

$$
\Psi_{31}=\int_{0}^{\pi / 2} d \theta \sin (\theta) \alpha_{3}\left(1+\beta_{1} \alpha_{2}+\beta_{2} \alpha_{1}-\beta_{1} \beta_{2}\right) / D .
$$

The equation for the magnon temperature has a more complicated form, namely

$$
F\left(T_{m}\right)=\frac{\Phi_{31} F\left(T_{H}\right)+\Psi_{13} F\left(T_{B}\right)}{\Phi_{31}+\Psi_{13}} .
$$

Here, indices $1 \leftrightarrow 3$ are swapped in $\Phi$ and $\Psi$, and $F(T)$ is a function of temperature that is determined by the integral

$$
F(T)=\int_{0}^{\infty} q^{3} v_{q f}(T) n_{q}(T) d q .
$$

In the case when the heating of the system is weak, i.e., $T_{H}-T_{B} \ll T_{B}$, the expression for the temperature difference between electrons and magnons is substantially simplified and can be written as

$$
T_{e}-T_{m}=\left(\frac{\Psi_{31}}{\Psi_{31}+\Phi_{13}}-\frac{\Phi_{31}}{\Phi_{31}+\Psi_{13}}\right)\left(T_{H}-T_{B}\right) .
$$

Dependence of $\left(T_{e}-T_{m}\right) /\left(T_{H}-T_{B}\right)$ on $T_{B} /\left(T_{H}-T_{B}\right)$ is shown in the Fig. 4. It is seen that for $T_{B} \gg T_{H}-T_{B}$ this

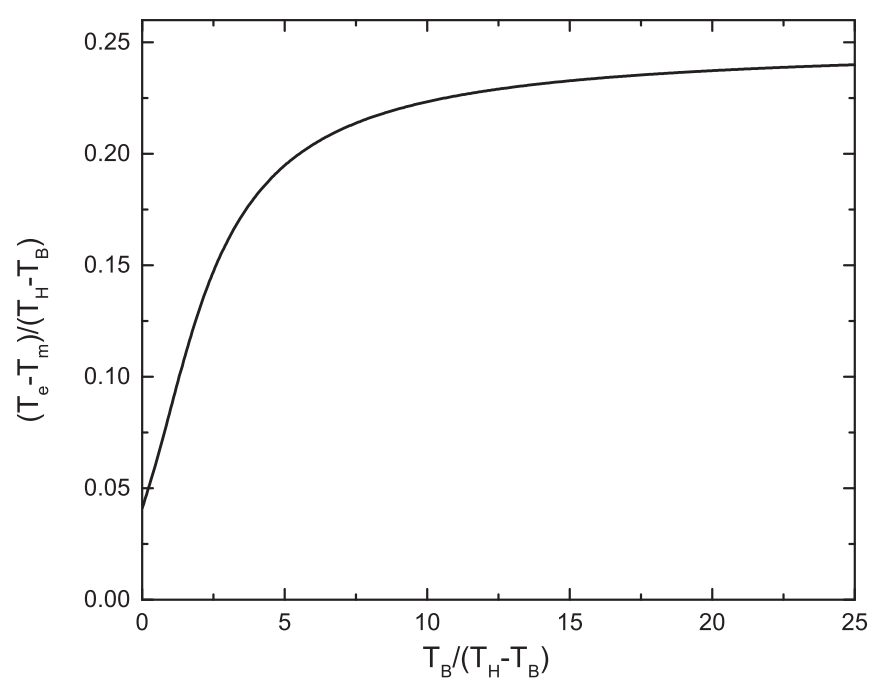

FIG. 4. The dependence of the difference between the electron and magnon temperatures on the thermostat temperature for thin $F$ and $N$ layers. To not complicate the physical picture, we put $\alpha_{1}(\theta)=$ $\alpha_{2}(\theta)=\alpha_{3}(\theta)=\alpha(\theta)$. For $\alpha(\theta)$, the stepwise approximation is used: $\alpha(\theta)=1 / 2$ for $\theta<\theta_{c r}$ and $\alpha(\theta)=0$ for $\theta>\theta_{c r}$ (where $\theta_{c r}$ is the angle of total internal reflection) [33]. At large $T_{B} /\left(T_{H}-T_{B}\right)$, the curve approaches asymptotically to 0.25 .

dependence goes to a constant value, which is described by the Eq. (56).

As in the previous section, we consider the case of $d_{f} \gg l_{f}, d_{n} \ll l_{n}$, which is important for the experiment. In this case, the determinant $D=e^{2 d_{f} / l_{f}}\left(1-\beta_{2} \beta_{3}\right)$, and the equations for the magnon and electron temperatures have the form

$$
\begin{gathered}
\int_{q_{z}>0} \frac{d^{3} q}{(2 \pi)^{3}} \hbar \omega_{q} s_{f z}\left\{\alpha_{1}\left[n_{q}\left(T_{m}\right)-n_{q}\left(T_{B}\right)\right]\right. \\
\left.-\frac{\alpha_{2} \alpha_{3}}{\left(1-\beta_{2} \beta_{3}\right)}\left[n_{q}\left(T_{H}\right)-n_{q}\left(T_{m}\right)\right]\right\}=0 \\
\int_{q_{z}>0} \frac{d^{3} q}{(2 \pi)^{3}} \frac{\hbar \omega_{q} v_{n q}}{\left(1-\beta_{2} \beta_{3}\right)}\left\{\alpha_{2}\left(1+\beta_{3}\right)\left[n_{q}\left(T_{e}\right)-n_{q}\left(T_{m}\right)\right]\right. \\
\left.-\alpha_{3}\left(1+\beta_{2}\right)\left[n_{q}\left(T_{H}\right)-n_{q}\left(T_{e}\right)\right]\right\}=0 .
\end{gathered}
$$

In both equations, the integration over the angles of the phonon wave vector is separated from the integration over its absolute value. As a result of the integration, we obtain for the magnon temperature

$$
\left\langle\alpha_{1}\right\rangle\left(T_{m}^{4}-T_{B}^{4}\right)=\left\langle\frac{\alpha_{2} \alpha_{3}}{1-\beta_{2} \beta_{3}}\right\rangle\left(T_{H}^{4}-T_{m}^{4}\right),
$$

and Eq. (58) gives

$$
T_{e}^{5}=\frac{1}{2}\left[T_{m}^{5} \frac{\overline{\alpha_{2}\left(1+\beta_{3}\right)}}{1-\beta_{2} \beta_{3}}+T_{H}^{5} \frac{\overline{\alpha_{2}\left(1+\beta_{3}\right)}}{1-\beta_{2} \beta_{3}}\right],
$$

where we have denoted

$$
\overline{f(\theta)}=\int_{0}^{\pi / 2} \sin (\theta) f(\theta) d \theta .
$$


For weak heating, when $T_{H}-T_{B} \ll T_{B}$, the difference between the electron and magnon temperatures is given by

$$
T_{e}-T_{m}=\frac{1}{2} \frac{\overline{\alpha_{3}\left(1+\beta_{2}\right)}}{1-\beta_{2} \beta_{3}} \frac{\left\langle\alpha_{1}\right\rangle\left(T_{H}-T_{B}\right)}{\left\langle\alpha_{1}\right\rangle+\left\langle\alpha_{2} \alpha_{3} /\left(1-\beta_{2} \beta_{3}\right)\right\rangle} .
$$

Thus, we have obtained expressions for the electron and magnon temperatures for two different sets of experiments: (i) when in a heterostructure "normal metal-ferromagnetic insulator-dielectric substrate," the metal layer is heated by a direct current $[19,20]$ and (ii) when the "normal metalferromagnetic dielectric" bilayer is enclosed between two massive dielectric plates that are maintained at different temperatures.

\section{COMPARISON WITH EXPERIMENT}

Recall that within the framework of the considered model, the only mechanism for creating a spin current is the temperature difference between magnons and electrons on the $F / N$ interface. Thus, if $T_{e} \neq T_{m}$, the magnon gas is not in equilibrium with the electron gas and the magnon absorption by electrons is not compensated by their emission. As a consequence, the interaction of nonequilibrium magnons with electrons leads to spin polarization of the electron gas near the $F / N$ interface and to the subsequent diffusion of spinpolarized electrons into the $N$ layer, i.e., to the spin current $\mathbf{J}_{s}$. As a result of the spin-orbit interaction, the spin current generates a charge current in the perpendicular direction and a potential difference $V_{\text {ISHE }}$ emerges at the lateral edges of the $N$ layer. When the electron and magnon temperature difference is small in comparison with the substrate temperature, the value $V_{\mathrm{ISHE}} \propto\left(T_{e}-T_{m}\right)[17,24,32,34,35]$. The direct proportionality between $V_{\mathrm{ISHE}}$ and $\left(T_{e}-T_{m}\right)$ allows us to compare our results with experiment.

From the results of Sec. II, it follows that for a continuous heating of the $N$ layer, the difference between the electron and magnon temperatures is proportional to the specific heating power $W$, and hence $V_{\mathrm{ISHE}} \propto W$. This linear dependence was observed in the experiment $[19,20]$. In the case when the $F / N$ system is enclosed between two heat reservoirs with temperatures $T_{H}$ and $T_{B}$, and $T_{H}>T_{B}$, the value $V_{\text {ISHE }} \propto$ $\left(T_{H}-T_{B}\right)$ (see the experimental works $\left.[7,36,37]\right)$. According to the results of Sec. III, the temperature difference $T_{e}-T_{m}$ is proportional to $T_{H}-T_{B}$ [see Eq. (56)]. Thus, in the case of the $I_{1} / F / N / I_{2}$ sandwich, the results of our theory agree with the experiment. Also, the dependence $T_{e}-T_{m}$ on $T_{B}$, presented in Fig. 4, agrees with the previous calculations [38].

We note an interesting feature that is predicted in Sec. II, namely, the growth of $T_{e}-T_{m}$ (and therefore, also $V_{\mathrm{ISHE}}$ ) when the temperature of the thermostat $T_{B}$ decreases at the fixed heating power $W$. The reason for this growth is an increase in the thermal resistance of the interfaces when the temperature is lowered. This behavior of $V_{\mathrm{ISHE}}\left(T_{B}\right)$ was observed in the experiments [16,24].

According to Fig. 2, at the same temperature of the thermostat $T_{B}$, the difference between the electron and magnon temperatures $\left(T_{e}-T_{m}\right)$ is smaller for thicker $F$ layers. This result agrees with the experiment [21], where a decrease in the electric field strength in the $N$ layer was observed when the thickness of the $F$ layer increased from $100 \mathrm{~nm}$ to 1 $\mu \mathrm{m}$. Also, in the experiment from Ref. [21], $V_{\mathrm{ISHE}}$ growth was observed for thin $F$ layers at $T_{B} \rightarrow 0$. This behavior of $V_{\mathrm{ISHE}}$ is consistent with the dependence $\left(T_{e}-T_{m}\right)$ on $T_{B}$, represented by curve 2 in Fig. 2. So, at low $T_{B}$, curve 1 agrees with experiment on the bulk sample, and curve 2 agrees with experiments on thin layers of YIG. In the case of heating of the $N$ layer, the dependence of $T_{e}-T_{m}$ on the temperature of the thermostat (Fig. 2) differs qualitatively from the same dependence in the case of a fixed temperature difference $T_{H}-T_{B}$ (Fig. 4). This physical picture is consistent with the experimental results presented in Fig. 1 in Ref. [21] and in Fig. 6 in Ref. [37].

As can be seen from Fig. 2 here and from Fig. 1 in Ref. [21], the discrepancy between the results of the theory and experiment takes place at high temperatures $T_{B}$, when in the theory $\left(T_{e}-T_{m}\right) \rightarrow 0$, and in the experiment $V_{\mathrm{ISHE}} \rightarrow$ const. In our opinion, this difference is due to the fact that for large $T_{B}$, the spin current is mainly generated by the temperature gradient in the $F$ layer. Since the temperature gradient is not taken into account in our theory, its range of applicability is limited to the low-temperature region.

\section{CONCLUSIONS}

Based on the microscopic approach, we theoretically analyzed the temperature and thickness dependencies of the voltage of the ISHE $V_{\text {ISHE }}$ for $N / F / I$ and $I_{1} / N / F / I_{2}$ heterostructures. We assumed that at low temperatures the voltage $V_{\mathrm{ISHE}}$ is proportional to the difference between the electron and magnon temperatures at the $N / F$ interface. We calculated this temperature difference and showed that the theoretical dependence of $V_{\text {ISHE }}$ on the bath temperature $T_{B}$ has a qualitatively different form for the following experimental situations. In the case when the $N / F$ bilayer is enclosed between two dielectric plates $I_{1}$ and $I_{2}$ having different temperatures, the dependence $V_{\text {ISHE }}\left(T_{B}\right)$ increases monotonically with $T_{B}$. If the $N / F / I$ heterostructure is heated by the current flowing in the $N$ layer, the dependence $V_{\text {ISHE }}\left(T_{B}\right)$ is decreasing. These theoretical results are consistent with the low temperature experiment. At high temperatures, the predictions of our theory differ from experiment, and we associate this discrepancy with the contribution to the spin current of the magnon temperature gradient, which is neglected in the theory. An analysis of the case of the Joule heating of the $N / F / I$ heterostructure also implies that as the thickness of the $F$ layer increases, the voltage $V_{\text {ISHE }}$ decreases. We showed that this behavior is determined by the ratio between the thickness of the $F$ layer and the length of phonon-magnon collisions, since in the thick $F$ layer the magnons are heated due to interaction with nonequilibrium phonons.

\section{ACKNOWLEDGMENTS}

The research leading to these results has received funding from the European Union's Horizon 2020 research and innovation program under Marie Sklodowska-Curie Grant Agreement No. 644348 (MagIC). 


\section{APPENDIX A: THE COEFFICIENTS $C_{i}^{\gtrless}$}

Substitution of the solutions Eqs. (14) and (15) into the boundary conditions (9), (11)-(13) yields a system of linear equations for the four coefficients $C_{i}^{\gtrless}$. The solution of the system is

$$
\begin{aligned}
C_{f}^{<}\left(\mathbf{q}_{f}^{\prime}\right)= & \frac{1}{D}\left\{\alpha _ { 1 } [ ( 1 - 2 \alpha _ { 2 } ) - \beta _ { 2 } e ^ { 2 d _ { n } / l _ { n } } ] \left[n_{q}\left(T_{m}\right)\right.\right. \\
& \left.-n_{q}\left(T_{B}\right)\right]+\alpha_{2} e^{d_{f} / l_{f}}\left(e^{2 d_{n} / l_{n}}-1\right) \\
& \left.\times\left[n_{q}\left(T_{e}\right)-n_{q}\left(T_{m}\right)\right]\right\} \\
C_{n}^{<}\left(\mathbf{q}_{n}^{\prime}\right)=- & \frac{\alpha_{2}}{D}\left\{\alpha_{1}\left[n_{q}\left(T_{m}\right)-n_{q}\left(T_{B}\right)\right]\right. \\
& \left.+\left(e^{d_{f} / l_{f}}-\beta_{1} e^{-d_{f} / l_{f}}\right)\left[n_{q}\left(T_{e}\right)-n_{q}\left(T_{m}\right)\right]\right\} \\
C_{f}^{>}\left(\mathbf{q}_{f}\right)= & \frac{e^{d_{f} / l_{f}}}{D}\left\{\beta_{1} \alpha_{2}\left(e^{2 d_{n} / l_{n}}-1\right)\left[n_{q}\left(T_{e}\right)-n_{q}\left(T_{m}\right)\right]\right. \\
& \left.-\alpha_{1} e^{d_{f} / l_{f}}\left(e^{2 d_{n} / l_{n}}-\beta_{2}\right)\left[n_{q}\left(T_{m}\right)-n_{q}\left(T_{B}\right)\right]\right\} \\
C_{n}^{>}\left(\mathbf{q}^{\prime}{ }_{n}\right)=- & \frac{\alpha_{2}}{D} e^{2 d_{n} / l_{n}+d_{f} / l_{n}}\left\{\alpha _ { 1 } e ^ { d _ { f } / l _ { f } } \left[n_{q}\left(T_{m}\right)\right.\right. \\
- & \left.\left.n_{q}\left(T_{B}\right)\right]+\left(e^{2 d_{f} / l_{f}}-\beta_{1}\right)\left[n_{q}\left(T_{e}\right)-n_{q}\left(T_{m}\right)\right]\right\},
\end{aligned}
$$

where $D$ denotes the determinant of the system

$$
D=e^{2 d_{n} / l_{n}+2 d_{f} / l_{f}}-\beta_{1} \beta_{2} e^{2 d_{n} / l_{n}}-\beta_{2} e^{2 d_{f} / l_{f}}+\beta_{1}\left(1-2 \alpha_{2}\right) \text {. }
$$

For compactness of expressions, we renamed $\alpha_{f \rightarrow i}$ through $\alpha_{1}$, and $\alpha_{n \rightarrow f}$ through $\alpha_{2}$. In this case, $\beta_{i}=1-\alpha_{i} \quad(i=1,2)$.

In the case of the $N / F$ two-layer system located between two insulators with different temperatures, the expressions for the coefficients $C_{i}^{\gtrless}$ are as follows:

$$
\begin{aligned}
C_{f}^{<}\left(\mathbf{q}_{f}^{\prime}\right)= & \frac{1}{D}\left\{\alpha_{2} \alpha_{3} e^{d_{n} / l_{n}+d_{f} / l_{f}}\left[n_{q}\left(T_{H}\right)-n_{q}\left(T_{e}\right)\right]\right. \\
& +\alpha_{2} e^{d_{f} / l_{f}}\left(e^{2 d_{n} / l_{n}}-\beta_{3}\right)\left[n_{q}\left(T_{e}\right)-n_{q}\left(T_{m}\right)\right] \\
& -\alpha_{1}\left[\beta_{2} e^{2 d_{n} / l_{n}}-\left(\beta_{2}-\alpha_{2}\right) \beta_{3}\right] \\
& \left.\times\left[n_{q}\left(T_{m}\right)-n_{q}\left(T_{B}\right)\right]\right\}, \\
C_{n}^{<}\left(\mathbf{q}^{\prime}{ }_{n}\right)= & \frac{e^{-d_{f} / l_{f}}}{D}\left\{\alpha_{3} e^{d_{n} / l_{n}+d_{f} / l_{f}}\left(e^{d_{f} / l_{f}}-\beta_{1} \beta_{2} e^{-d_{f} / l_{f}}\right)\right. \\
& \times\left[n_{q}\left(T_{H}\right)-n_{q}\left(T_{e}\right)\right]-\alpha_{2} \beta_{3}\left(e^{2 d_{f} / l_{f}}-\beta_{1}\right)\left[n_{q}\left(T_{e}\right)\right. \\
- & \left.\left.n_{q}\left(T_{m}\right)\right]-\alpha_{1} \alpha_{2} \beta_{3} e^{d_{f} / l_{f}}\left[n_{q}\left(T_{m}\right)-n_{q}\left(T_{B}\right)\right]\right\},
\end{aligned}
$$

$$
\begin{aligned}
C_{f}^{>}\left(\mathbf{q}_{f}\right)= & \frac{e^{d_{f} / l_{f}}}{D}\left\{\beta_{1} \alpha_{2} \alpha_{3} e^{d_{n} / l_{n}}\left[n_{q}\left(T_{H}\right)-n_{q}\left(T_{e}\right)\right]\right. \\
& +\beta_{1} \alpha_{2}\left(e^{2 d_{n} / l_{n}}-\beta_{3}\right)\left[n_{q}\left(T_{e}\right)-n_{q}\left(T_{m}\right)\right] \\
& \left.-\alpha_{1} e^{d_{f} / l_{f}}\left(e^{2 d_{n} / l_{n}}-\beta_{2} \beta_{3}\right)\left[n_{q}\left(T_{m}\right)-n_{q}\left(T_{B}\right)\right]\right\},
\end{aligned}
$$

$$
\begin{aligned}
C_{n}^{<}\left(\mathbf{q}_{n}^{\prime}\right)= & \frac{e^{d_{n} / l_{n}+d_{f} / l_{n}}}{D}\left\{\alpha_{3}\left[e^{2 d_{f} / l_{f}}-\beta_{1}\left(\beta_{2}-\alpha_{2}\right)\right]\right. \\
& \times\left[n_{q}\left(T_{H}\right)-n_{q}\left(T_{e}\right)\right]-\alpha_{2} e^{d_{n} / l_{n}}\left(e^{2 d_{f} / l_{f}}-\beta_{1}\right) \\
& \times\left[n_{q}\left(T_{e}\right)-n_{q}\left(T_{m}\right)\right]-\alpha_{1} \alpha_{2} e^{d_{n} / l_{n}+d_{f} / l_{n}} \\
& \left.\times\left[n_{q}\left(T_{m}\right)-n_{q}\left(T_{B}\right)\right]\right\}
\end{aligned}
$$

where

$$
\begin{aligned}
D= & e^{2 d_{n} / l_{n}+2 d_{f} / l_{f}}-\beta_{1} \beta_{2} e^{2 d_{n} / l_{n}}-\beta_{2} \beta_{3} e^{2 d_{f} / l_{f}} \\
& +\beta_{1}\left(1-2 \alpha_{2}\right) \beta_{3}
\end{aligned}
$$

\section{APPENDIX B: MODEL VALIDITY}

Let us briefly discuss conditions of applicability of our model, which operates with concepts of electron and magnon temperatures. As noticed earlier [31,39], the electron temperature can be introduced if the thermalization time of electrons, i.e., the time of electron-electron collisions $\tau_{e e}$, is smaller than the electron-phonon energy relaxation time $\tau_{e}$. In pure metals for $T_{e} \ll \Theta_{D}$, this inequality holds if $T_{e} \lesssim k_{B} \Theta_{D}^{2} / \varepsilon_{F} \sim 1 \mathrm{~K}$. In dirty films, the condition $\tau_{e e}<\tau_{e}$ is satisfied in a wider region $T_{e} \lesssim 10 \mathrm{~K}$ [40]. At high temperatures, the electrons will be thermalized if their temperature $T_{e}>\Theta_{D}\left(\varepsilon_{F} / k_{B} \Theta_{D}\right)^{1 / 3}$, that is, $T_{e} \gtrsim 10^{3} \mathrm{~K}$.

Unlike $T_{e}$, the conditions necessary for introducing the magnon temperature are much less stringent. The condition necessary for introducing the magnon temperature is that the magnon-magnon collision frequency is much larger than the collision frequency of magnons with phonons. For fourmagnon processes, the average frequency of magnon-magnon collisions [41] is

$$
v_{m m} \sim \frac{\Theta_{C}}{\hbar}\left(\frac{T}{\Theta_{C}}\right)^{4}
$$

The average frequency of magnon-phonon collisions is

$$
v_{m p}(T)=18.2 v_{0}\left(\frac{\Theta_{D}}{\Theta_{C}}\right)^{1 / 2}\left(\frac{T}{\Theta_{D}}\right)^{5 / 2} \exp \left(-\frac{\Theta_{D}^{2}}{4 \Theta_{C} T}\right)
$$

where

$$
v_{0}=\frac{15 \Theta_{D}}{32 \pi^{5} M_{f} a_{f} s_{f}}\left(\frac{\Theta_{D}}{\Theta_{C}}\right)^{2}
$$

The value $\left(v_{m p}\right)^{-1}$ is equal to the characteristic time of the energy relaxation of magnons on phonons $\tau_{m p}$ [42]. A comparison of Eq. (B1) with Eq. (B2) shows that for $\Theta_{D} \sim \Theta_{C}$ (as in YIG) the magnon-magnon collision frequency is significantly higher than the collision frequency of magnons with phonons for any $T$ and the magnon temperature can be introduced at all thermostat temperatures $T_{B}$. 
[1] G. E. W. Bauer, A. H. MacDonald, and S. Maekawa, Solid State Commun. 150, 459 (2010).

[2] G. E. W. Bauer, Spin caloritronics, in Spin Current, edited by S. Maekawa, S. O. Valenzuela, E. Saitoh, T. Kimura (Oxford University Press, Oxford, England, 2012).

[3] G. E. W. Bauer, E. Saitoh, and B. J. Van Wees, Nat. Mater. 11, 391 (2012).

[4] S. R. Boona, R. C. Myers, and J. P. Heremans, Energy Environ. Sci. 7, 885 (2014).

[5] K. Uchida, S. Takahashi, K. Hari, J. Ieda, W. Kishibae, K. Ando, S. Maekawa, and E. Saitoh, Nature 455, 778 (2008).

[6] K. Uchida, J. Xiao, H. Adachi, J. Ohe, S. Takahashi, J. Ieda, T. Ota, Y. Kajiwara, H. Umezawa, H. Kawai, G. E. W. Bauer, S. Maekawa, and E. Saitoh, Nat. Mater. 9, 894 (2010).

[7] K. Uchida, T. Nonaka, T. Ota, and E. Saitoh, Appl. Phys. Lett. 97, 262504 (2010).

[8] C. M. Jaworski, J. Yang, S. Mack, D. D. Awschalom, J. P. Heremans, and R. C. Myers, Nat. Mater. 9, 898 (2010).

[9] M. Schreier, F. Kramer, H. Huebl, S. Geprags, R. Gross, S. T. B. Goennenwein, T. Noack, T. Langner, A. A. Serga, B. Hillebrands, and V. I. Vasyuchka, Phys. Rev. B 93, 224430 (2016).

[10] E. Saitoh, M. Ueda, H. Miyajima, and G. Tatara, Appl. Phys. Lett. 88, 182509 (2006).

[11] S. O. Valenzuela and M. Tinkham, Nature (London) 442, 176 (2006).

[12] T. Kimura, Y. Otani, T. Sato, S. Takahashi, and S. Maekawa, Phys. Rev. Lett. 98, 156601 (2007).

[13] L. Vila, T. Kimura, and Y. C. Otani, Phys. Rev. Lett. 99, 226604 (2007).

[14] T. Seki, Y. Hasegawa, S. Mitani, S. Takahashi, H. Imamura, S. Maekawa, J. Nitta, and K. Takanashi, Nat. Mater. 7, 125 (2008).

[15] K. Uchida, H. Adachi, T. Ota, H. Nakayama, S. Maekawa, and E. Saitoh, Appl. Phys. Lett. 97, 172505 (2010).

[16] E.-J. Guo, J. Cramer, A. Kehlberger, C. A. Ferguson, D. A. MacLaren, G. Jakob, and M. Klaui, Phys. Rev. X 6, 031012 (2016).

[17] M. Schreier, A. Kamra, M. Weiler, J. Xiao, G. E. W. Bauer, R. Gross, and S. T. B. Goennenwein, Phys. Rev. B 88, 094410 (2013).

[18] S. Y. Huang, W. G. Wang, S. F. Lee, J. Kwo, and C. L. Chien, Phys. Rev. Lett. 107, 216604 (2011).

[19] M. Schreier, N. Roschewsky, E. Dobler, S. Meyer, H. Huebl, R. Gross, and S. T. B. Goennenwein, Appl. Phys. Lett. 103, 242404 (2013).
[20] W. X. Wang, S. H. Wang, L. K. Zou, J. W. Cai, Z. G. Sun, and J. R. Sun, Appl. Phys. Lett. 105, 182403 (2014).

[21] A. Prakash, B. Flebus, J. Brangham, F. Yang, Y. Tserkovnyak, and J. P. Heremans, Phys. Rev. B 97, 020408(R) (2018).

[22] A. W. Little, Can. J. Phys. 37, 334 (1959).

[23] A. I. Akhieser and L. A. Shishkin, Zh. Eksp. Teor. Fiz. 34, 1267 (1958) [Sov. Phys. JETP 34, 875 (1958)].

[24] J. Kimling, G.-M. Choi, J. T. Brangham, T. Matalla-Wagner, T. Huebner, T. Kuschel, F. Yang, and D. G. Cahill, Phys. Rev. Lett. 118, 057201 (2017).

[25] V. A. Shklovskij, V. V. Kruglyak, R. V. Vovk, and O. V. Dobrovolskiy, Phys. Rev. B 98, 224403 (2018).

[26] A. I. Bezuglyj and V. A. Shklovskij, Fiz. Nizk. Temp. 39, 459 (2013); Low Temp. Phys. 39, 357 (2013).

[27] A. I. Bezuglyj and V. A. Shklovskij, Phys. Rev. B 89, 214303 (2014).

[28] S. B. Kaplan, J. Low Temp. Phys. 37, 343 (1979).

[29] E. T. Swartz and R. O. Pohl, Rev. Mod. Phys. 61, 605 (1989).

[30] V. A. Shklovsky, Zh. Eksp. Teor. Fiz. 78, 1281 (1980) [Sov. Phys. JETP 51, 646 (1980)].

[31] A. I. Bezuglyı̆ and V. A. Shklovskiŭ, Zh. Eksp. Teor. Fiz. 111, 2106 (1997) [JETP 84, 1149 (1997)].

[32] J. Xiao, G. E. W. Bauer, K.-c. Uchida, E. Saitoh, and S. Maekawa, Phys. Rev. B 81, 214418 (2010).

[33] A. I. Bezuglyj and V. A. Shklovskij, J. Phys.: Condens. Matter 30, 295001 (2018).

[34] H. Adachi, K. Uchida, E. Saitoh, and S. Maekawa, Rep. Prog. Phys. 76, 036501 (2013).

[35] S. A. Bender and Y. Tserkovnyak, Phys. Rev. B 91, 140402(R) (2015).

[36] T. Kikkawa, K. Uchida, Y. Shiomi, Z. Qiu, D. Hou, D. Tian, H. Nakayama, X.-F. Jin, and E. Saitoh, Phys. Rev. Lett. 110, 067207 (2013).

[37] S. M. Rezende, R. L. Rodriguez-Suarez, R. O. Cunha, A. R. Rodrigues, F. L. A. Machado, G. A. Fonseca Guerra, J. C. Lopez Ortiz, and A. Azevedo, Phys. Rev. B 89, 014416 (2014).

[38] Y. H. Shen, X. S. Wang, and X. R. Wang, Phys. Rev. B 94, 014403 (2016).

[39] A. I. Bezuglyj and V. A. Shklovskij, Fiz. Nizk. Temp. 42, 809 (2016); Low Temp. Phys. 42, 636 (2016).

[40] E. M. Gershenzon, M. E. Gershenzon, G. N. Gol'tsman, A. D. Semenov, and A. V. Sergeev, Pis'ma Zh. Eksp. Teor. Fiz. 36, 241 (1982) [JETP Lett. 36, 296 (1982)].

[41] A. I. Akhiezer, V. G. Bar'yakhtar, and S. V. Peletminskii, Spin Waves (North Holland, Amsterdam, 1968).

[42] A. I. Bezuglyj, V. A. Shklovskij, V. V. Kruglyak, and R. V. Vovk (unpublished). 